\title{
Image Threshold Selection Exploiting Empirical Mode Decomposition
}

\author{
Stelios Krinidis and Michail Krinidis \\ Information Management Department \\ Technological Institute of Kavala, \\ Ag. Loukas, 65404 Kavala, Greece \\ stelios.krinidis@mycosmos.gr, mkrinidi@gmail.com
}

\begin{abstract}
Thresholding process is a fundamental image processing method. Typical thresholding methods are based on partitioning pixels in an image into two clusters. A new thresholding method is presented, in this paper. The main contribution of the proposed approach is the detection of an optimal image threshold exploiting the empirical mode decomposition (EMD) algorithm. The EMD algorithm can decompose any nonlinear and non-stationary data into a number of intrinsic mode functions (IMFs). When the image is decomposed by empirical mode decomposition (EMD), the intermediate IMFs of the image histogram have very good characteristics on image thresholding. The experimental results are provided to show the effectiveness of the proposed threshold selection method.
\end{abstract}

Keywords: Threshold selection, clustering, empirical mode decomposition, ensemble empirical mode decomposition, intrinsic mode.

\section{Introduction}

Image thresholding is one of the main and most important tasks in image analysis and computer vision. Thresholding principle is based on distinguishing an object from the background in order to extract useful information from the image.

A large number of image thresholding techniques have been proposed in the literature [1]. However, the design of a robust and an efficient thresholding algorithm is far from being a simple process, due to the existence of images depicting complex scenes at low resolution, uneven illumination, scale changes, etc.

Thresholding techniques could be categorized in six groups [1] according to information being exploited. These categories are:

- Shape-based methods, which analyze the shape of the image histogram (i.e., the peaks, valleys and curvature). Each method uses different forms of shape properties, such as distance from the histogram convex hull [2], autoregressive modelling [3], overlapping peaks, etc.

- Clustering-based methods, which label the gray-level samples as background or foreground (object), or alternatively they model them as a mixture of two Gaussians [4 8]. In this category, the gray-level data undergoes a clustering analysis, with the number of clusters being always equal to two.

L. Iliadis et al. (Eds.): AIAI 2012, IFIP AICT 381, pp. 395 403, 2012.

(C) IFIP International Federation for Information Processing 2012 
- Entropy-based methods, which use the entropy of the foreground and the background regions, the cross-entropy between the original and the binarized image etc. [9, 10]. These algorithms exploit the entropy of the distribution of the gray levels in an image, by maximizing the entropy of the thresholded image or by minimizing the cross entropy.

- Attribute-based methods, which seek a measure between the gray-level and the binarized images, such as fuzzy shape similarity, edge coincidence, etc. [1, 12]. These algorithms evaluate the threshold value by using attributes quality or similarity measures between the initial gray-level image and the output binary image, such as edge matching, shape compactness, gray level moments, connectivity, texture or stability of segments objects [12], or fuzzy measures [11], etc.

- Spatial methods, which exploit higher-order probability distribution and/or correlation between the image pixels [13, 14]. The algorithms in this category utilize not only the gray level distribution, but also the dependency of pixels in a neighborhood, for example, the probabilities, correlation functions, cooccurrence probabilities, local linear dependence models of image pixels, 2-D entropy, etc.

- Local methods, which adapt the threshold value on each image pixel to the local image characteristics, such as range, variance [15-17], contrast, surfacefitting parameters of the pixel neighborhoods, etc.

This paper presents a novel, fast and robust image thresholding method. The method is based on the decomposition of the image histogram by the Empirical Mode Decomposition (EMD) [18] to its Intrinsic Mode Functions (IMFs). More specific, the decomposition is performed by the Ensemble Empirical Mode Decomposition (EEMD) [19], which provides noise resistance and assistance to data analysis. The properties of the desired IMFs [18, 19] will be shown that provide an efficient threshold for the image under examination.

The remainder of the paper is organized as follows. The thresholding method is introduced in Section 2, Experimental results are shown in Section 3 and conclusions are drawn in Section 4 .

\section{Threshold Selection Based-On EEMD}

In this Section, the image threshold selection method is introduced. This method is fully automated and is based on the IMFs extracted by the EEMD [19] algorithm applied on the histogram of the image under examination. More details regarding the Ensemble Empirical Mode Decomposition (EEMD) and the derived Intrinsic Mode Functions (IMFs), their properties and all the adopted assumptions are presented in [18, 19].

The histogram $h(k)$ is computed for an input image $I$ with $k=0 \ldots G$ and $G$ being the maximum luminance value in the image $I$, typically equal to 255 when 8-bit quantization is assumed. Then, the probability mass function of the image histogram is defined as the normalized histogram by the total pixel number:

$$
p(k)=\frac{h(k)}{N}
$$



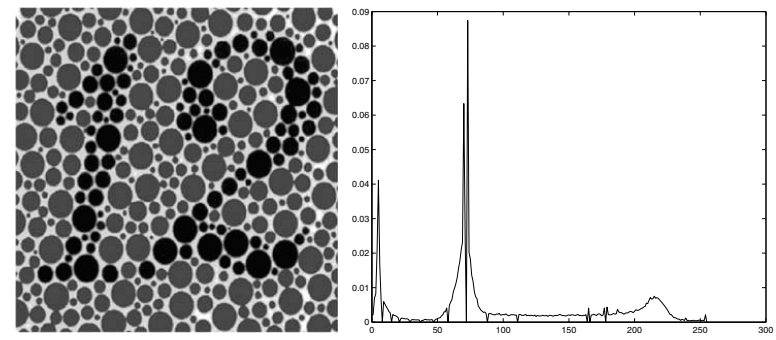

Fig. 1. An image for color blindness test and its normalized histogram

where $N$ is the total number of image pixels. An example of an image and its normalized histogram is depicted in Figure 1

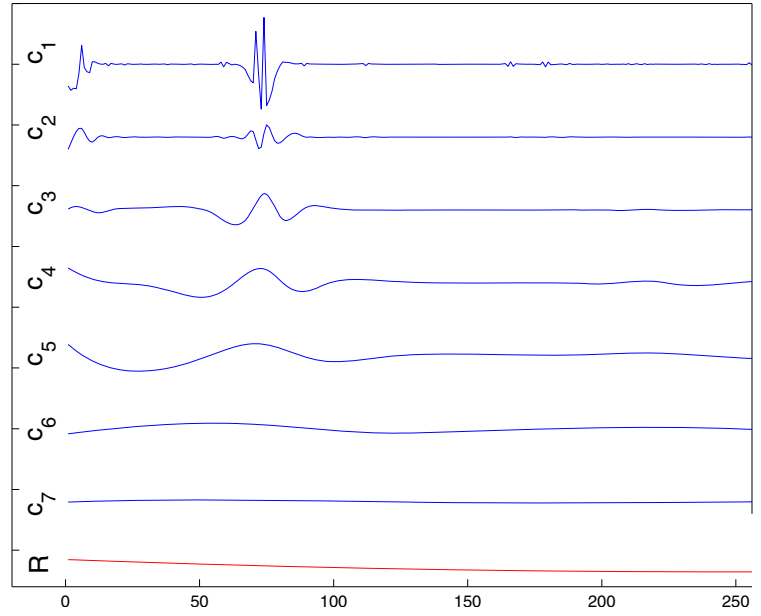

Fig. 2. The IMFs of the histogram for the image depicted in Figure 1 with a Gaussian noise of amplitude 0.2 and 1000 trials are performed

In the sequence, the proposed method analyzes the normalized histogram $p(k)$ of an image into its intrinsic mode functions (IMFs) exploiting the ensemble empirical mode decomposition (EEMD) algorithm. The IMFs of the histogram of the image shown in Figure1, are presented in Figure 2. The IMFs are produced using the EEMD algorithm with a Gaussian noise of amplitude equal to 0.2 and 1000 trials. The number of the extracted IMFs (including the residue function) for a 8 -bit quantized image is $\log _{2}(256)=8$.

One can easily notice in Figure 2 that the first IMF $c_{1}$ mainly carries the histogram "noise", irregularities and the sharp details of the histogram, while IMFs $c_{6}, c_{7}$ and the residue $R$ mostly describe the trend of the histogram. On the other hand, IMFs $c_{2}$ to $c_{5}$ describe the initial histogram with simple and 


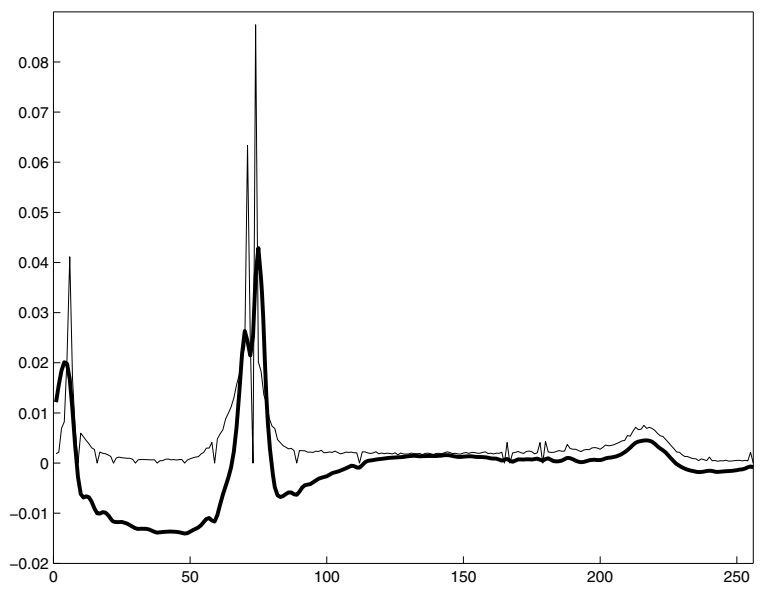

Fig. 3. The histogram of the image shown in Figure 1 (thin line) and the summation of the $c_{2}$ to $c_{5}$ IMFs (fat line)

uniform pulses. This is the main reason that the proposed method is focused on $c_{2}$ to $c_{5}$ IMFs. Let us now define the summation $c_{m}$ of these IMFs as follows:

$$
c_{m}=\sum_{i=2}^{5} c_{i} .
$$

Figure 3 depicts the summation $c_{m}$ (fat line) in contrast to the initial normalized histogram (thin line). One can notice that this summation $c_{m}$ describes the main part of the histogram leaving out all its meaningless details.

The minimum of summation $c_{m}$ is given by:

$$
T^{*}=\arg \left\{\min _{0 \leq T \leq G} c_{m}(T)\right\},
$$

where $T^{*}$ is the desired image threshold. Since the summation $c_{m}$ provides a better, more clear and uniform formation of the image histogram, its minimum can be considered as an optimal threshold for the input image and its efficiency will be experimentally shown in the next Section.

Finally, the overall algorithm could be summarized in Figure 4 .

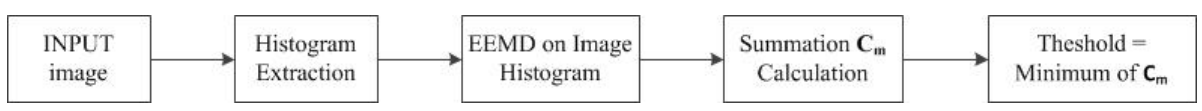

Fig. 4. The histogram of the image shown in Figure 1 (thin line) and the summation of the $c_{2}$ to $c_{5}$ IMFs (fat line) 


\section{Experimental Results}

In this Section, the performance of the proposed method is examined by presenting numerical results using the introduced thresholding approach on various synthetic and real images, with different types of histogram. The obtained results are compared with the corresponding results of four well-known thresholding methods [4 6, 11]. In all the experiments, the EEMD algorithm was used with a noise of amplitude equal to 0.2 and 1000 trials are performed.

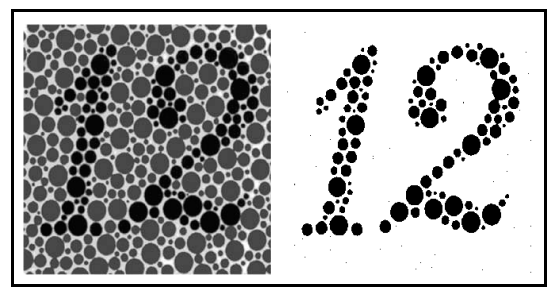

(a)

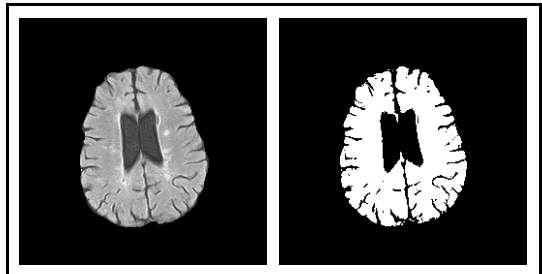

(b)

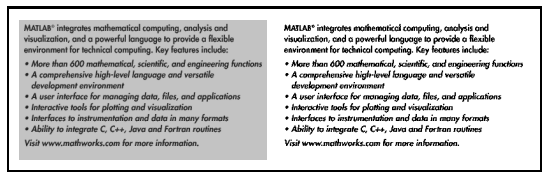

(c)

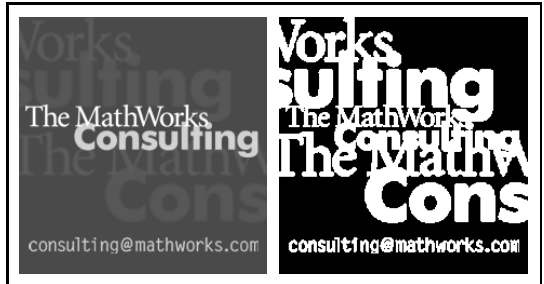

(d)

Fig. 5. Various images (left column) and their corresponding thresholded images produced by the proposed method (right column)

Figure 5 presents various real and synthetic images and their corresponding thresholded images obtained by the proposed approach. The left column shows the initial images, while the right column depicts the corresponding thresholded images produced by the proposed algorithm. One can clearly see that the proposed method can efficiently threshold the images under examination. Table 1 confirms the results in terms of the well known Tanimoto/Jaccard error [20] $E(\cdot)$ defined here as:

$$
E(o, m)=1-\frac{\int_{I_{o} \cap I_{m}} d x d y}{\int_{I_{o} \cup I_{m}} d x d y},
$$

where $I_{m}$ and $I_{o}$ are the extracted and the desired thresholded images respectively. In Table 1 the desired thresholded images have been extracted manually and then, compared (4) with the acquired thresholded images produced by the proposed method and four well known thresholding methods [4 6, 11]. The errors of the proposed methods are small enough to enforce one to claim that they 

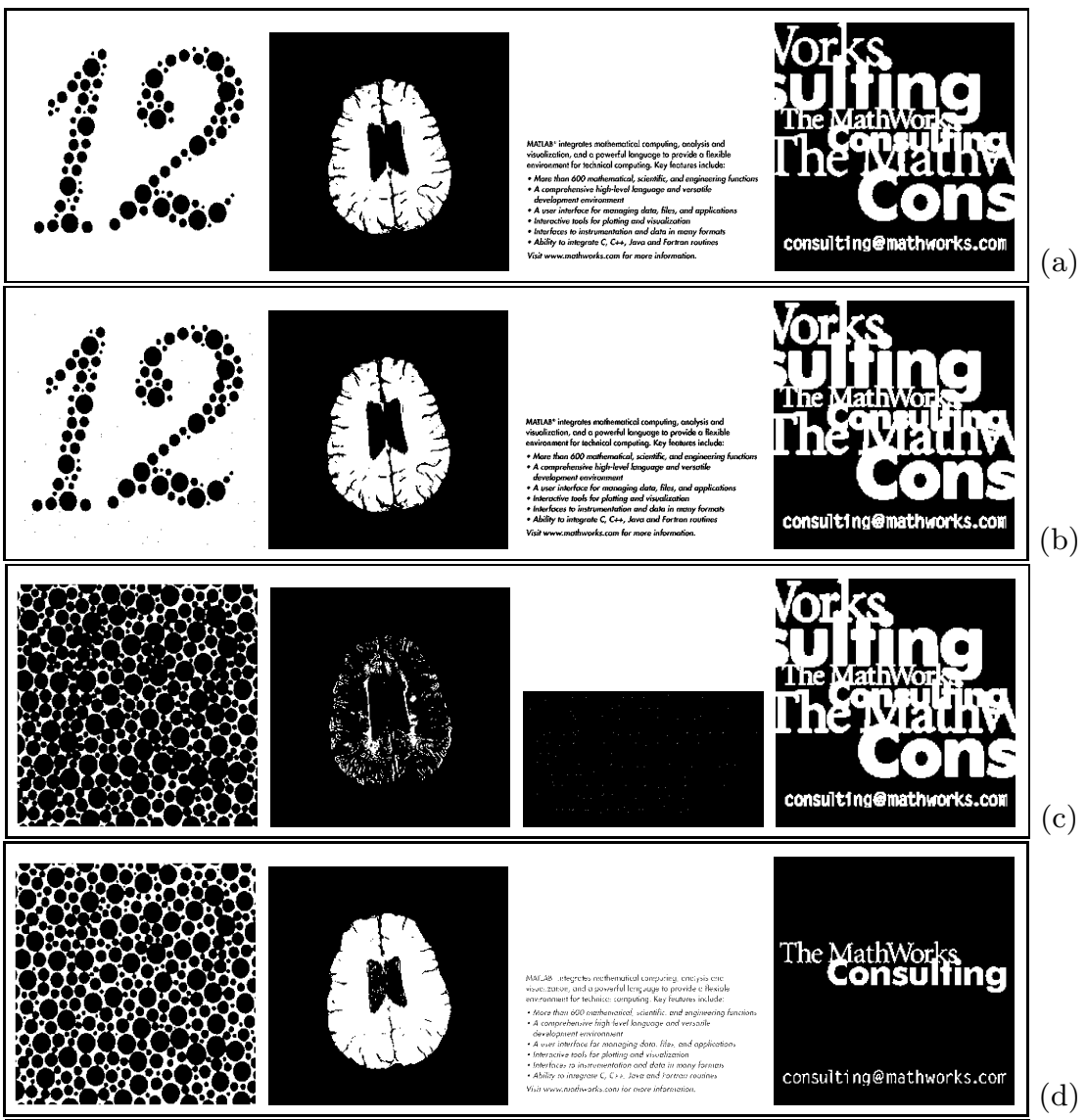

(d)
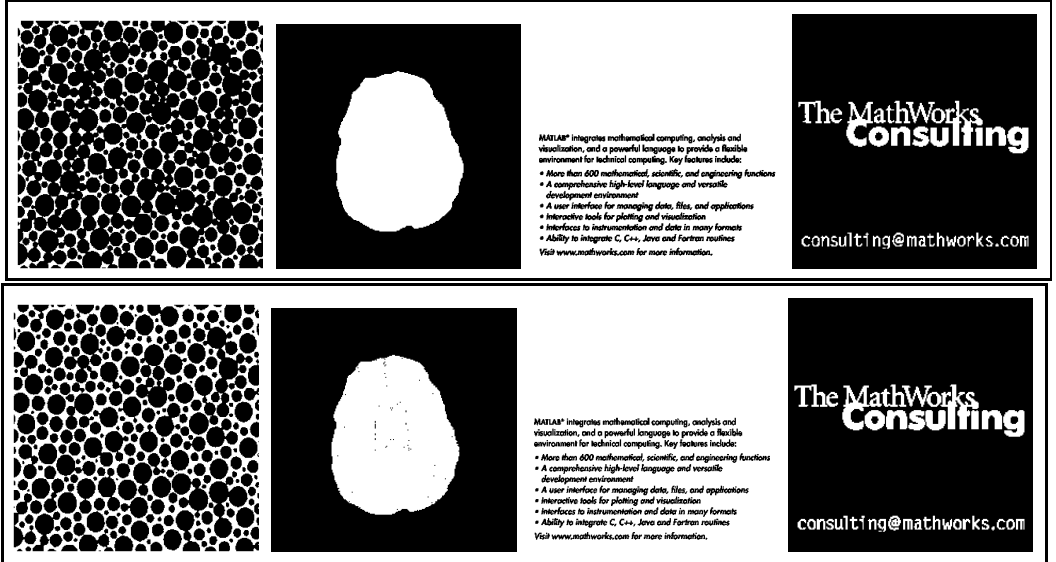

Fig. 6. Thresholded images: (a) ground truth, (b) proposed method, (c) Kittler's method, (d) Otsu's method, (e) Huang's method and (f) Kwon's method 
are insignificant. On the contrary, the other four methods produce larger errors, a fact that is also depicted in Figure 6. The thresholded images produced by the proposed algorithm are more efficient. In Table 1, is also shown, the thresholds produced by the corresponding algorithms (the value in the parenthesis).

Table 1. Threshold values determined by five threshold selection methods with the corresponding area difference measure results

\begin{tabular}{|c|c|c|c|c|}
\hline$\overline{\text { Method }}$ & $\begin{array}{l}\text { Color blindsness } \\
(\text { Fig. } 5(\mathrm{a}))\end{array}$ & $\begin{array}{l}\text { MRI image } \\
\text { (Fig. } 5 \text { (b)) }\end{array}$ & $\begin{array}{l}\text { Doc. image } \\
\text { (Fig. } 5(\mathrm{c}))\end{array}$ & $\begin{array}{c}1 \text { Doc. image } 2 \\
(\text { Fig. } 5(\mathrm{~d}))\end{array}$ \\
\hline$\overline{\overline{\text { Proposed }}}$ & $0.006(46)$ & $0.006(135)$ & 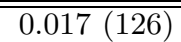 & $0.136(75)$ \\
\hline Kittler's [4] & $0.738(179)$ & $0.834(193)$ & 0.998 (195) & $0.136(75)$ \\
\hline Otsu's [6] & $0.615(136)$ & $0.100(86)$ & $0.091(45)$ & $0.781(140)$ \\
\hline Huang's [11] & $0.711(169)$ & $0.230(1)$ & $0.085(170)$ & $0.760(122)$ \\
\hline Kwon's [5] & $0.577(110)$ & $0.228(48)$ & $0.063(152)$ & $0.730(101)$ \\
\hline
\end{tabular}

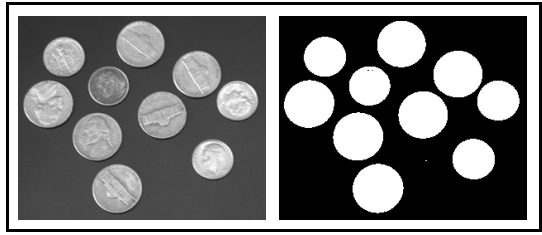

(a)

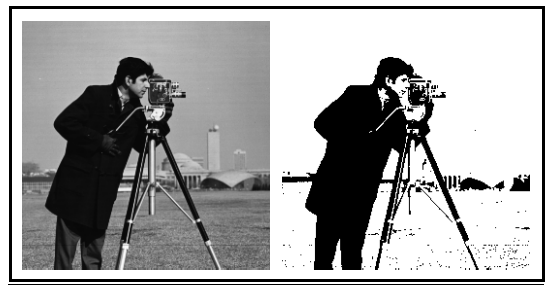

(b)
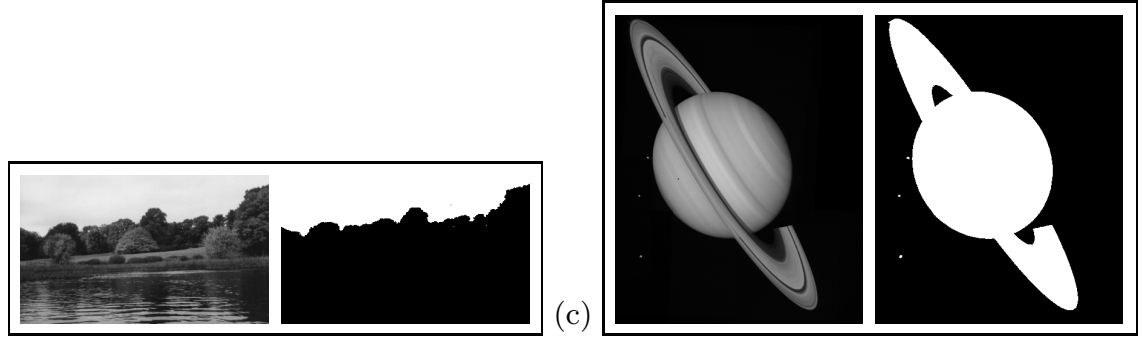

$(d)$

Fig. 7. Various images (left column) and their corresponding thresholded images produced by the proposed method (right column)

Figure 6 presents the thresholded images extracted by the proposed and the rest algorithms [4 6, 11]. Figure [6a shows the ground truth which manually extracted in order to calculate the numerical results depicted in Table 1 . Figure 6b depicts the thresholded images produced by the proposed algorithm, while Figures 6-6: show the thresholded images acquired by the Kittler's method [4] (Fig. 6c), Otsu's method [6] (Fig. 6d), Huang's method [11] (Fig.6p) and Kwon's method [5] (Fig. 6f).

Finally, Figure 7 shows various images (left column) and their corresponding thresholded images acquired by the proposed method. All images in Figure 7 
depict complex scenes, especially Figure 7(d). However, the proposed algorithm thresholds these images in a very reasonable way, a fact that is also proved in Figures 5 and 6 and in parallel provides better performance than the other four methods.

However, the algorithm fails to threshold an image correctly when the desired cluster is too small.

\section{Conclusion}

In this paper, a novel image thresholding method is introduced. The proposed approach exploits ensemble empirical mode decomposition (EEMD) to analyze the normalized histogram of the image under examination into a number of intrinsic mode functions (IMFs). The proposed algorithm uses only specific components, the intermediate IMFs of the EEMD decomposition, in order to evaluate an optimal image threshold. The effectiveness of the proposed threshold selection method is proved in the experimental results Section where the proposed thresholding algorithm is applied to various images with simple and complex scenes.

\section{References}

1. Sezgin, M., Sankur, B.: Survey over image thresholding techniques and quantitative performance evaluation. Journal of Electronic Imaging 13(1), 146-165 (2004)

2. Sahasrabudhe, S., Gupta, K.: A valley-seeking threshold selection technique. In: Computer Vision and Image Understanding, pp. 55-65 (1992)

3. Guo, R., Pandit, S.: Automatic threshold selection based on histogram modes and a discriminant criterion. Machine Vision and Applications 10(5-6), 331-338 (1998)

4. Kittler, J., Illingworth, J.: Minimum error thresholding. Pattern Recognition 19(1), 41-47 (1986)

5. Kwon, S.: Threshold selection based on cluster analysis. Pattern Recognition Letters 25(9), 1045-1050 (2004)

6. Otsu, N.: A threshold selection method from gray level histograms. IEEE Transactions on Systems, Man, and Cybernetics 9(1), 62-66 (1979)

7. Krinidis, M., Pitas, I.: Color texture segmentation based on the modal energy of deformable surfaces. IEEE Transactions on Image Processing 18(7), 1613-1622 (2009)

8. Krinidis, S., Chatzis, V.: Fuzzy energy-based active contours. IEEE Transactions on Image Processing 18(12), 2747-2755 (2009)

9. Sahoo, P., Wilkins, C., Yeaget, J.: Threshold selection using renyi's entropy. Pattern Recognition 30(1), 71-84 (1997)

10. Yen, J., Chang, F., Chang, S.: A new criterion for automatic multilevel thresholding. IEEE Transactions on Image Processing 4(3), 370-378 (1995)

11. Huang, L., Wang, M.: Image thresholding by minimizing the measures of fuzziness. Pattern Recognition 28(1), 41-51 (1995)

12. Pikaz, A., Averbuch, A.: Digital image thresholding based on topological stable state. Pattern Recognition 29(5), 829-843 (1996)

13. Cheng, H., Chen, Y.Y.: Fuzzy partition of two-dimensional histogram and its application to thresholding. Pattern Recognition 32(5), 825-843 (1999) 
14. Krinidis, S., Chatzis, V.: A robust fuzzy local information c-means clustering algorithm. IEEE Transactions on Image Processing 19(5), 1328-1337 (2010)

15. Sauvola, J., Pietikainen, M.: Adaptive document image binarization. Pattern Recognition 33(2), 225-236 (2000)

16. Aghagolzadeh, M., Soltanian-Zadeh, H., Araabi, B., Aghagolzadeh, A.: A hierarchical clustering based on mutual information maximization. In: IEEE International Conference on Image Processing, vol. I, pp. 277-280 (September 2007)

17. Yu, F.X., Lei, Y.Q., Wang, Y.G., Lu, Z.M.: Robust image hashing based on statistical invariance of dct coefficients. Journal of Information Hiding and Multimedia Signal Processing 1(4), 286-291 (2010)

18. Huang, N., Shen, Z., Long, S., Wu, M., Shih, E., Zheng, Q., Tung, C., Liu, H.: The empirical mode decomposition method and the Hilbert spectrum for nonstationary time series analysis. Proceedings of the Royal Society of London 454, 903-995 (1998)

19. Wu, Z., Huang, N.: Ensemble empirical mode decomposition: A noise-assisted data analysis method. Advances in Adaptive Data Analysis 1(1), 1-41 (2009)

20. Tohka, J.: Surface Extraction from Volumetric Images Using Deformable Meshes: A Comparative Study. In: Heyden, A., Sparr, G., Nielsen, M., Johansen, P. (eds.) ECCV 2002, Part III. LNCS, vol. 2352, pp. 350-364. Springer, Heidelberg (2002) 